\title{
Sense of Time Continuity: Possible Mechanisms of Disruption in Schizophrenia
}

\author{
Anne Giersch*, Laurence Lalanne*, Mitsouko van Assche*, \\ Patrick E. Poncelet*, and Mark A. Elliott**†
}

\section{Introduction}

Several psychiatrists have described clinical symptoms that suggest a disturbed sense of time continuity (i.e., a fragmentation of time) in patients with schizophrenia (Fuchs, 2007; Vogeley and Kupke, 2007). All aspects of mental life involve the succession of events in time, and according to several authors (Andreasen, 1999; Minkowski, 1933), time disturbance might play a central role in the pathophysiology of disorders such as schizophrenia. Approaching the phenomenological experience of time in patients with schizophrenia is complex, particularly given patients' difficulty in reporting their inner mental life. Patients nonetheless report disturbances that can be taken as hints as to how they experience their disorder. For example, Chapman (1966) describes several patients' reports as follows:

Things go too quick for my mind. [... It's as if you were seeing one picture one minute and another picture the next.

You are dying from moment to moment and living from moment to moment and you're different each time.

When I start walking I get a fast series of pictures in front of me. Everything seems to change and revolves around me. Something goes wrong with my eyes and I've got to stop and to stand still.

What are the mechanisms underlying the sense of time continuity in healthy adults and what type of impairment leads to the disturbances experienced by patients? On a more practical level, how can we address questions of the

\footnotetext{
* INSER U1114; Dept of Psychiatry; University Hospital of Strasbourg; Fédération de Médecine Translationnelle de Strasbourg (FMTS); 1, pl de l'Hôpital; 670oo Strasbourg.

** Department of Psychology; National University of Ireland, Galway, University Road, Galway, Ireland.

$\dagger$ Department of Human Sciences, Faculty of Design, Kyushu University, Fukuoka, Japan.

(C) ANNE GIERSCH ET AL., 2015 | DOI 10.1163/9789004230699_006

This is an open access chapter distributed under the terms of the Creative Commons
} 
experience of time continuity using an experimental approach and objectify clinical descriptions? These are questions that are fundamental to the work described in the following paragraphs. Mechanisms involved in the sense of time continuity may operate at various time scales (reviewed in Wittmann, 2011). In our work, we have chosen to focus on very short time scales below $100 \mathrm{~ms}$, with the aim of assessing the elementary mechanisms that subtend processing of successive stimulus events in patients. A focus on short time scales was motivated by several results from the literature: elementary timing mechanisms have been related directly with neuronal synchronization (reviews in Elliott, 2005; Elliott et al., 2006; van Wassenhove, 2009), which seems appropriate given that schizophrenia is very precisely characterized by abnormalities in synchronization at frequencies in the EEG gamma band (i.e., at around $40 \mathrm{~Hz}$ ), and, thus, correspond to intervals of around $25 \mathrm{~ms}$ (reviewed in Uhlhaas and Singer, 2010). In addition, impairments are also observed at a behavioural level that may be explained by elementary time disorders, such as in motor actions (Carroll et al., 2009; Delevoye-Turrell et al., 2003): for example, when participants lift an object or hit a target with an object, the peak grip force is usually synchronized with the time of impact or maximal load. In patients, however, grip force is delayed by around 30 to 10oms (Delevoye-Turrell et al., 2003). This means a difficulty at precisely synchronizing grip force in time. Another example of abnormalities at short time scales can be found in visual perception tasks: it has been proposed that patients have difficulties to efficiently allocate attention in time (i.e., to focus attention at precise moments in time), when two stimuli follow each other at delays of between $5^{0}$ to $250 \mathrm{~ms}$ Stimulus Onset Asynchrony (soA; Granholm et al., 2009; Lalanne et al., 2012a). Impairments in timing at the shortest time scales have all the more to be considered in schizophrenia that these time scales might be involved in the sense of time continuity.

In fact, different time scales must be involved in the sense of time continuity: first, there is a lower margin in our ability to distinguish separate events in time. Even though two spatially separate stimuli are presented at different times for very brief intervals they may appear to be presented simultaneously. Exner (1875) suggested minimum time differences in temporal order discrimination for intervals of up to 17 and $44 \mathrm{~ms}$. Considering invariances, a common measure that extends across sensory modalities seems to be the minimum time required for temporal order discrimination following the successive presentation of more than two stimuli. For tactile and visual stimuli, and irrespective to the precise structure of the visual stimuli concerned, simultaneity thresholds have been determined with remarkably little variation: Brecher (1932) showed what he referred to as units of 'subjective time' corresponded to 
average periods of $55.3 \mathrm{~ms}$ for tactile stimulation and periods of $56.9 \mathrm{~ms}$ for visual stimulation, with standard deviations of no greater than $1.4 \mathrm{~ms}$.

This observation relates to the concept of a window of time, or perceptual moments, within which all events are processed together and perceived as occurring simultaneously (Brecher, 1932; Elliott et al., 2006, 2007; Lalanne, 1876; van Wassenhove, 2009; von Baer, 1864; Wittmann, 2011). Although referred to in terms of perception, time windows of this order of magnitude would represent an elementary quantum without experienced duration. Only larger time windows of up to 2 to $3 \mathrm{~s}$ would be associated with the experience of duration, and these would represent the second time scale involved in the sense of time continuity. At these larger time scales, information would not be temporally fused and as such perceived in terms of a perceptual unity but would be separate events grouped together within the same moment of experience. A popular example is the experience of present time arising when listening to a melody: when listening to the present tone, the previous tone is still in mind, while the coming tone is usually anticipated. Because past, present, and future tones are all momentarily present in mind, all of them are part of the subjective present. The past tone is nonetheless known as being past, and is thus both past (in objective time) and present (in subjective present time). Similar reasoning holds for the future tone, and this leads to the concept of specious present (James, 1896). Husserl (1893-1917) has proposed that the integration of past, present, and future represents a key mechanism in our sense of time continuity. It is not known to which amount and how the shorter 3oms time windows are integrated within the experienced 3-s moments (Pöppel, 1997, 2009; Szelag et al., 2004). This requires an understanding of how windows overlap and integrate with one another (Dainton, 2010; Elliott, 2005). It might be proposed that temporal windows and their overlap are brought about by neuronal synchronization (Varela, 1999). Because neuronal synchronization of action potentials requires time, even the processing of the simplest event, like the display of a square on a computer screen, is time-consuming, and is coded within a temporal window. Different events would then overlap in time even if their onset were shifted in time. The result would be a sense of continuity rather than the perception of discrete moments.

There are, thus, several candidate mechanisms bringing about a sense of time continuity, but a number of questions require clarification: are events judged to be simultaneous really processed as co-temporal? Is the overlap of events enough to bring about continuity of time, or is it required to integrate events with one another? At which time scale do these mechanisms occur? We first explored the length of the elementary time window in patients with schizophrenia. We found these elementary time windows to be altered in 
patients, but these disturbances led us to wonder about the processing of events within time windows. To answer this question, we devised a new method of analyzing participants' responses. The results question the usual assumption that events are treated as co-temporal within temporal windows, and lead us to reconsider the mechanisms underlying the sense of time continuity. We summarize our findings in the following. ${ }^{1}$

Measuring the Windows of Visual Simultaneity

Visual stimuli separated in time by an SoA of less than 20 to $30 \mathrm{~ms}$ are judged as being simultaneous. Our aim was to compare the associated time window in patients with schizophrenia against that found in healthy participants. The paradigm used to determine this window is relatively simple: two visual stimuli (two bars or two squares, for instance) are shown on a computer screen. They appear either simultaneously or with a short asynchrony and participants decide whether the two stimuli are simultaneous or asynchronous. In our experiments, participants gave their response by pressing on a left response key in case of simultaneity or a right response key in case of an asynchrony. To date, four studies have shown that patients need larger asynchronies than healthy participants to report two stimuli as appearing at different times (Foucher et al., 2007; Giersch et al., 2009; Lalanne et al., 2012b; Schmidt et al., 2011). Several possible confounds have been eliminated, i.e. the role of eye movements, interhemispheric transfer, modality specificity, bias, and access to consciousness. Hereafter, we review shortly how these possibilities have been discarded.

It has been shown that the impairment persists if patients are required to look at a central fixation point until the stimuli appear (Lalanne et al., 2012b) indicating that the impairment is not related to abnormal eye movements (Holzman, 2000; Phillips and Davis, 1994; Trillenberg et al., 2004). In addition, the deficit is similar if stimuli are displayed within the same hemifield or across hemifields (Lalanne et al., 2012b), thus eliminating a possible role for impaired

1 Our groups of patients with schizophrenia (around 20 in each study) were mildly symptomatic outpatients, usually treated with antipsychotics, but without benzodiazepines. Their performance was compared with those of control participants individually matched on sex, age, and education level (Giersch et al., 2009; Lalanne et al., 2012a,b,c). Until now, no result allows us to firmly exclude an effect of antipsychotic medication, although the doses of antipsychotic medication do not correlate with performance. It is to be noted that clinical descriptions of time continuity disruptions predate the establishment of antipsychotic medication. Besides, the mechanisms of the sense of time continuity and its impairments need to be unraveled even in treated patients. 
interhemispheric connections (Knöchel et al., 2012; Mohr et al., 2008; Schwartz et al., 1984; but see David, 1993). A difficulty at discriminating simultaneous from asynchronous events is observed also in auditory (Foucher et al., 2007) and cross-modal conditions (audio-visual; Martin et al., 2013). Finally, a possible decisional bias has been carefully discarded (Giersch et al., 2009; Schmidt et al., 2011). A decisional bias is independent of perceptual ability and may occur during response selection: in this case it might be that patients process asynchronies as do the healthy participants but need larger asynchronies to select an 'asynchronous' response. Classical methods based upon signal detection theory (Green and Swets, 1974) showed no difference between patients and healthy participants regarding bias (Giersch et al., 2009; Schmidt et al., 2011).

We used an additional method, based upon priming, which bypasses the problem of an explicit judgment on the part of the patients. The possibility of impairment due to the explicit nature of the response is distinct from a decisional bias. A deficit at making explicit judgment is related to the mechanisms allowing information to become conscious (Del Cul et al., 2006), whereas decisional biases are related to variations in the use of information to give a response. Each time a patient is explicitly asked to make a judgment, and show abnormal performance, it can be asked whether the impairment is related to the difficulty the patients experience in formulating an explicit judgment. Patients have often been shown to be thus impaired although implicit information processing remains unimpaired (Del Cul et al., 2006). In the present context, a seeming difficulty to detect an asynchrony between two items might be due to a non-specific difficulty at making a subjective judgment, rather than impairment in processing the timing of events. In our task, we used a procedure developed by Elliott et al. (2007) and took an implicit measure of the effects of an asynchrony on a subsequent explicit judgment of simultaneity/ asynchrony. In this paradigm, two priming bars were displayed on a computer screen, either simultaneously or asynchronously while a series of 6 distracter bars were rapidly switched on and off around the priming bars, thus making the temporal relation between the priming bars impossible to accurately report. After the distracters switched off the priming bars remained on screen and after a short interval increased in luminance separately and with a variable SOA (which included a simultaneous increase; Figure 5.1). Participants reported whether this luminance increase was simultaneous or asynchronous across the two bars.

Elliott et al. (2007) showed that in healthy volunteers, the masked asynchronous bars biased participants towards reporting an asynchrony in the subsequent increase in bar luminance. We showed, in addition, that this bias increases with the asynchrony of the priming bars: the larger the prime's 


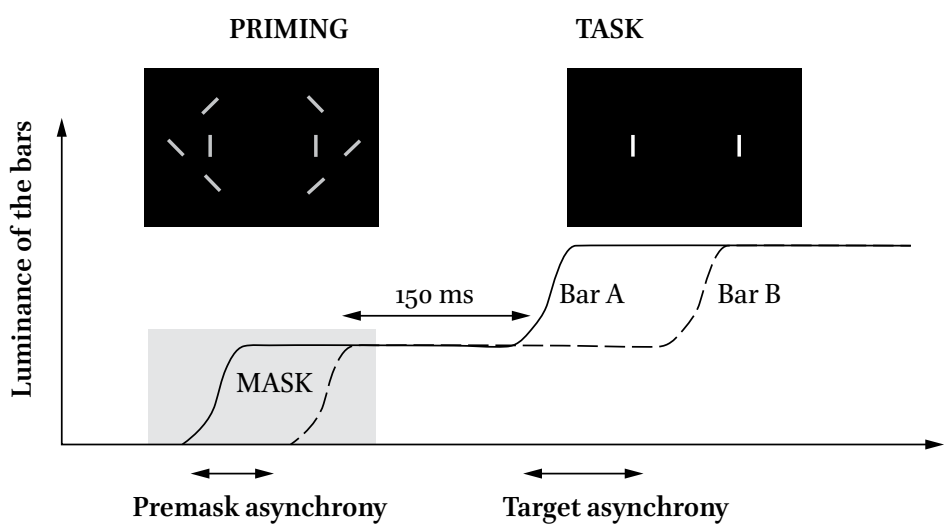

FIGURE 5.1 Illustration of the paradigm used to check for a non-specific effect of subjective judgments. The curves represent the increase in luminance of the two target bars, $A$ and $B$. The first increase in luminance is used as a prime and masked by the distracters ('priming'figure). The prime is asynchronous when the two bars increase their luminance asynchronously. The participant's task is to decide whether the second increase in luminance is simultaneous or asynchronous. With the permission of schizophrenia bulletin.

asynchrony, the larger the bias (Giersch et al., 2009). This was observed even though the prime's asynchrony was individually adapted so that it was below threshold in all cases, indicating that the effect is due to the implicit priming of the asynchrony. We reasoned that if the difficulties of the patients are due to impaired explicit judgments per se, there should be a dissociation between implicit and explicit processing of the asynchrony: explicit judgments of asynchrony would be impaired while their implicit influence would remain unaffected. If this were the case, we expected priming to be identical between groups for equivalent sub-threshold asynchronies. This is not what was observed, however: similar priming effects were observed in the two groups, but for larger asynchronies in patients than in healthy participants. This suggests that the enlarged temporal window observed in patients reveals a true difficulty at discriminating events over time, and is not explicable in terms of a non-specific difficulty in formulating the required judgment.

Inasmuch as the subjective experience of the present is built upon elementary time windows, it can be expected that a lengthened interval of subjective simultaneity distort the sense of time continuity. Before drawing this conclusion however, we questioned whether this lengthened interval entailed the temporo-perceptual fusion of all the events within the associated temporal window, i.e., whether it meant that events are treated as co-temporal even at an implicit level. It was indeed surprising to observe the amplitude of the 
deficit in some patients with schizophrenia, who were unable to detect an asynchrony of more than 10oms. Did this really mean that patients fuse events in time over periods longer than 10oms? The next step was, thus, to check the integrity of implicit information processing within the window of simultaneity.

\section{Implicit Processing of Information within the Temporal Window}

In order to evaluate the implicit processing of information within the temporal window, we devised an original method of analysis, by examining the Simon effect. The Simon effect refers to the fact that participants are faster and more accurate when a visual stimulus is presented within the same perceptual hemifield as their responding hand (Hommel, 2011a). The precise mechanisms of the Simon effect are a matter of discussion (i.e., Hommel, 2011a,b; van der Lubbe and Abrahamse, 2011), but the important point is that the effect is independent of the explicit instructions given to the participant. In our paradigm, it allowed us to check whether or not the stimuli are fused in time. During our task, two stimuli are displayed on the screen, one to the left and one to the right and participants give their response - 'simultaneity' or 'asynchrony' by pressing the left or right response key, respectively. When the two stimuli are displayed simultaneously, a Simon effect cannot occur: the participants cannot be biased to respond on any particular side since the displayed information is equivalent to both sides. However, when the stimuli are asynchronous there is an asymmetry and under these conditions we observed a Simon effect (Lalanne et al., 2012b,c). Healthy participants were systematically biased to answer to the side of the second stimulus independent of its right or left location (Lalanne et al., 2012c). Given that physical information is identical on both sides, it is only the temporal difference between the two stimuli that can lead to such a Simon effect. The important point is that this Simon effect was observed even at short asynchronies when participants reported the majority of stimulus presentations to be simultaneous (Lalanne et al., 2012b,c). This result suggests that the asynchrony is processed even though participants are unable to report it. Interestingly, a Simon effect was also observed in patients with schizophrenia. At large asynchronies patients were biased to the side of the second stimulus to the same extent as the healthy participants (Lalanne, 2012b). However, at small asynchronies, patients were biased to the side of the first stimulus (Lalanne et al., 2012b,c). This effect has been observed in three different studies, with three different groups of 18 to 20 patients with schizophrenia. It has been observed for asynchronies of 8 to $17 \mathrm{~ms}$, i.e., at delays that 
lead to rates of 'simultaneous' responses identical to the rates observed for perfect synchrony (Lalanne, 2012c). As with healthy participants, this means that the asynchronies are processed in patients with schizophrenia even for delays of below 2oms and are not fused in time. Had the stimuli been fused in time, then the two stimuli would have been processed as if identical. In that case, the Simon effect could not have occurred, i.e., the subjects could not have been biased to either stimulus. The Simon effect shows that patient's process very short asynchronies even though they need much larger asynchronies than healthy participants to explicitly report them. These data show a clear dissociation between the implicit response - indicated by the Simon effect, and the subjective experience of temporal relations between stimuli - indicated by the explicit judgments of those relations. A similar dissociation has also been observed with multisensory stimuli (Martin et al., 2013). Our results however do not indicate a preserved implicit processing. On the contrary, the results in patients with schizophrenia differ qualitatively from those in healthy participants: at short asynchronies, the responses of healthy participants are biased to the side of the second stimulus whereas the responses of patients are biased to the side of the first stimulus. Hence, consistent with our previous studies (Giersch et al., 2009), it seems that both the implicit and explicit processing of asynchronies is affected in patients with schizophrenia.

The critical question at this stage is the meaning of the bias to the side of the first or second stimulus. The bias to the side of the second stimulus initially seems surprising, given the effect of prior entry. This effect has been demonstrated using similar tasks, involving simultaneity/asynchrony or temporal order judgments on successive or simultaneous stimuli (review in Spence and Parise, 2010). It has been frequently shown that cueing the first stimulus in a sequence of two facilitates subsequent judgments of order or simultaneity/ asynchrony. A cue, for example, an indicator or a flash, encourages the deployment of attention to the cued location. This is believed to speed up processing of the first stimulus, thus facilitating the detection of an asynchrony or of a succession between first and second stimuli (Spence and Parise, 2010). This shows that focusing attention on the first stimulus in a sequence is important for the processing of sequential order. By contrast, we found that the response of healthy participants was biased towards the second stimulus. The major differences between the two observations is, first, that our observations take place when subjects are not aware of any asynchrony, and second, that prior entry takes place before critical stimuli are presented. In contrast, the Simon effect is recorded at the time of the participant's response, i.e., after the display of the stimuli. From these observations it is tempting to conclude that healthy participants move from the first to the second stimulus during the sequence, 
as if biased towards the last occurring event. Conversely, patients would be unable to do so and would remain stuck on the first stimulus.

A number of difficulties and questions still remain with this interpretation: first, it might be possible that deployment of visuo-spatial attentional mechanisms rather than time disorders account for the difficulties of patients with schizophrenia. Our results suggest a form of move between the first and second stimulus. Given that the two stimuli differ not only on their time onset but also on their spatial location, it might be asked whether patients have a difficulty to move from the first to the second stimulus as the result of spatial or visual organization impairments. In fact, patients with schizophrenia are known to be impaired at grouping items when those items are spatially separate (review in Silverstein and Keane, 2011), and this might have made it difficult for them to sequentially process from the first to the second stimulus. To check for this possibility, we used the fact that patients' difficulties with perceptual grouping are alleviated when clear grouping cues relate the stimuli (Giersch and Rhein, 2008; van Assche and Giersch, 2011).We slightly adapted our paradigm and added line-segments between the stimuli in order to facilitate grouping (Figure 5.2).

We also manipulated the spatial predictability of the second stimulus to further facilitate the move towards this stimulus in patients: in one experiment there was only two possible locations for the stimuli and the second stimulus location was always predictable, whereas in a second experiment, 4 locations were used, and the location of the second stimulus was uncertain (Figure 5.3).

Since perceptual grouping promotes the perception of synchrony in time (Nicol and Shore, 2007), it was expected that all Simon effects would be reduced by making right and left stimuli more similar in their temporal properties. As expected, healthy participants' bias to the side of the second stimulus was reduced when stimuli were connected (Lalanne et al., 2012c). On the other hand, the Simon effect to the side of the second stimulus was increased when the location of the second stimulus was predictable. The results in healthy participants, therefore, confirmed that our experimental manipulations had the expected effect. However, in patients there was a very clear bias to the side of the first stimulus when the first and second stimulus were related with a linesegment, and when the second stimulus location was predictable in $100 \%$ of the cases (Figure 5.4).

This suggests that in patients the abnormal bias to the side of the first stimulus persists when grouping and spatial difficulties are alleviated by experimental manipulations. Overall, these results suggested difficulties related to time rather than to spatial and visual organization impairment. 


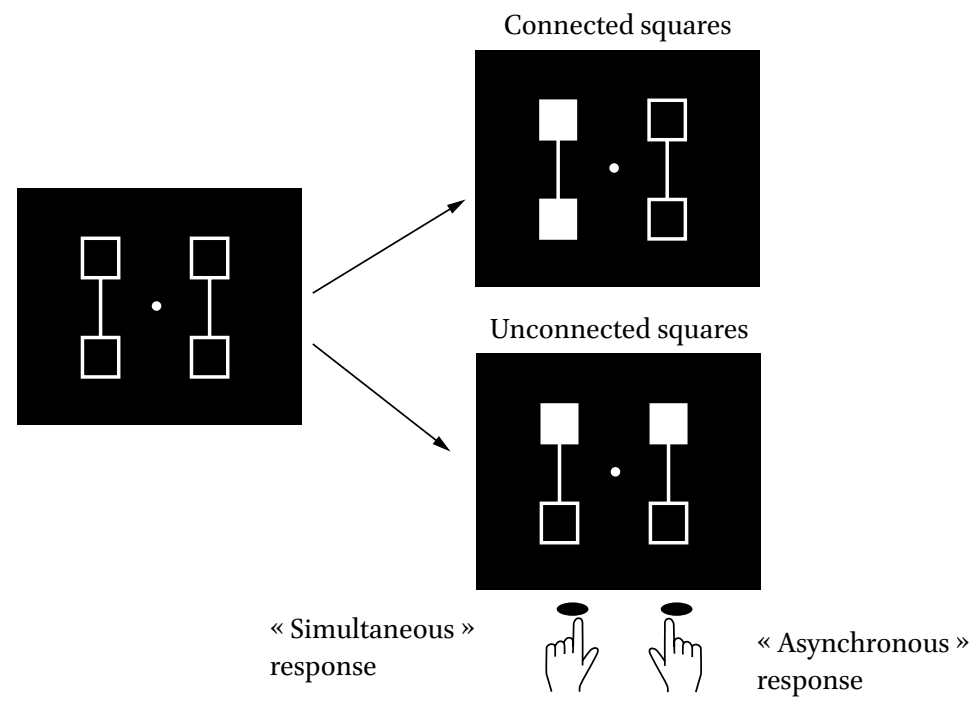

FIGURE 5.2 Illustration of the procedure designed to compare simultaneity/asynchrony discrimination for connected and unconnected squares and squares displayed in the same hemifield vs. in different hemifields. Two squares are filled in, in gray, either simultaneously or asynchronously. These two squares are either connected or not. Participants are instructed to hit the right key when they think the squares are filled in asynchronously and the left key when they think the filling occurs simultaneously. In the examples, filled squares are connected and located within the same hemifield in the right top figure and unconnected and in different hemifields in the right lower figures. The intra-vs. across-hemifield manipulation has been conducted without connecters in a first experiment (Lalanne et al., 2012b) and with connecters in a second, distinct experiment (Lalanne et al., 2012c). The examples correspond to the second experiment. In this experiment, the location of the connecters (vertical vs. horizontal) and the location of the targets (within the same vs. different hemifields) yielded four main possibilities (targets connected within the same or different hemifields and targets unconnected within the same or different hemifields), which were equally represented and displayed in random order.

A difficulty remained, however, in the interpretation of the Simon effect, because we do not know whether participants are coding a succession between the first and the second stimulus when the asynchronies are very short. This point is critical when considering the sense of time continuity. As emphasized above, it has been proposed that the sense of time continuity arises from the integration of past, present, and future moments within the subjective present. If such succession is coded within the most elementary time windows, integration would take place on a shorter time scale than previously believed. It would then have to be explained how events are coded one relative to another on time scales shorter than 2oms. Automatically ordering events in time might 

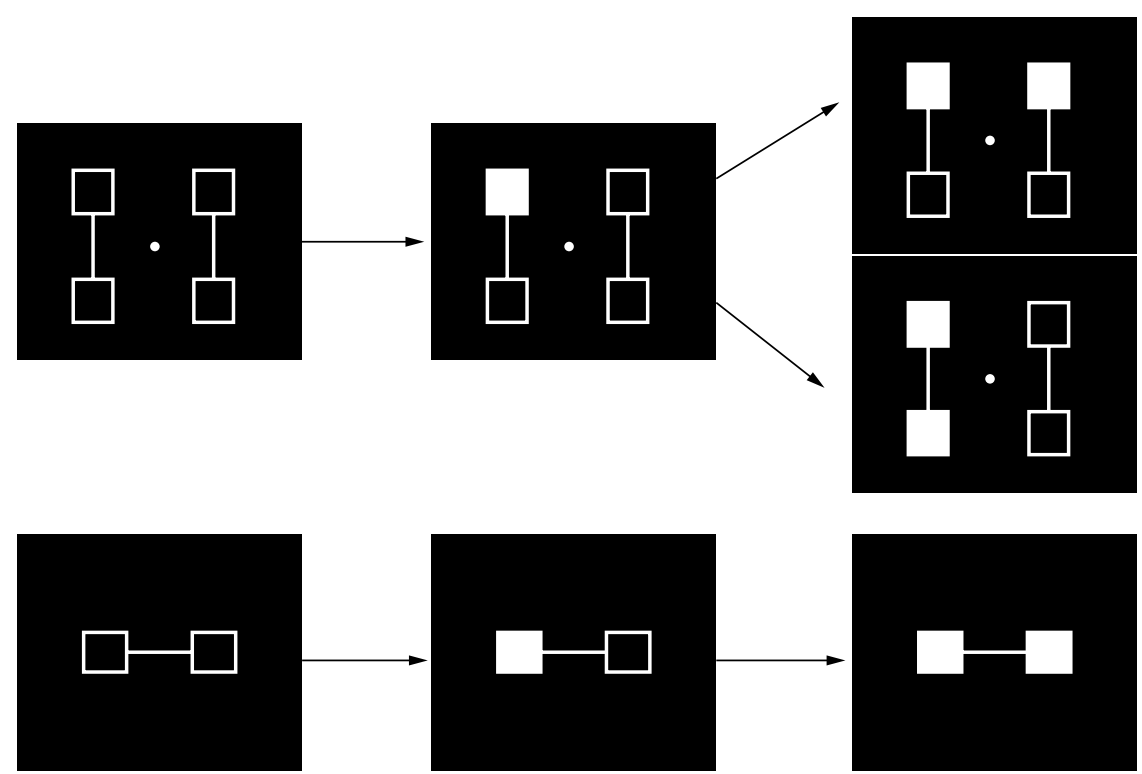

FIGURE 5.3 Illustration of the successive events in the simultaneity/asynchrony discrimination task in case of an asynchrony (from left to right). When 4 locations are used (upper row) and the first square is filled in (middle figure in the upper row), there are two possible locations for the second one (figure on the right), and there is, thus, an uncertainty regarding the location of the second stimulus. The spatial location of the second stimulus is always predictable when only two locations are used (lower row). These two experiments have been conducted in two different groups of participants (Lalanne et al., 2012c).

indeed be costly. In our experiments, stimuli were sometimes displayed in two different hemifields and, thus, processed in different cerebral hemispheres (cf. Figure 5.2). Comparing their onsets is bound to involve long-distance connectivity (at least to transfer information regarding the stimulus onset) and some specialized comparison mechanisms. If this is to be generalized in the natural environment, it would mean permanent comparisons between unrelated stimuli. The usefulness of such computation might be questioned. Beside, the computation cost would increase exponentially in a crowded environment. It might, thus, be proposed that even if events delayed by short asynchronies are not processed as being co-temporal, their succession is not coded automatically. This possibility is supported by our most recent results in healthy participants (Poncelet et al., in preparation). We used a priming paradigm during which two square frames (two empty squares) are displayed, one to the right and one to the left of fixation (Figure 5.5). These two priming frames are displayed either simultaneously or asynchronously, but the asynchrony is only 


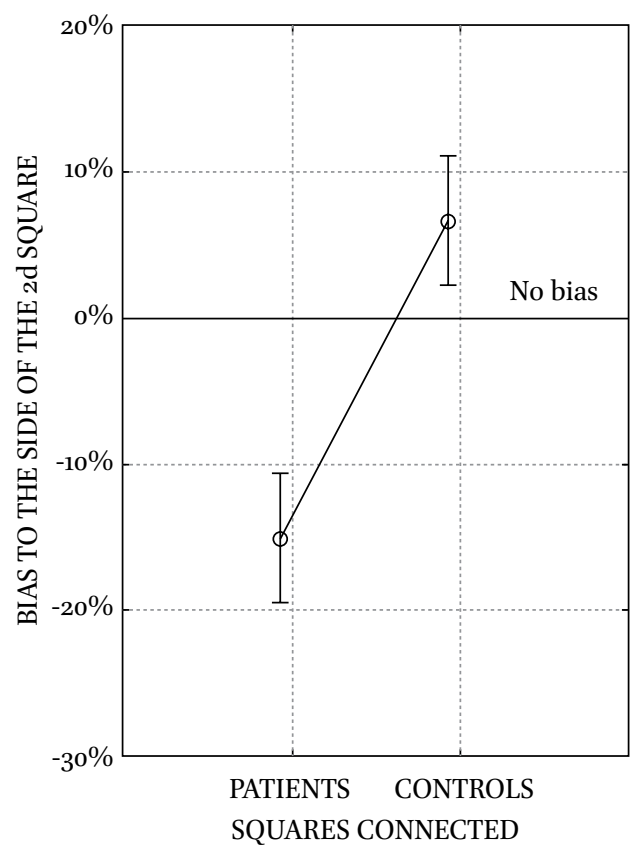

FIGURE 5.4 Amplitude of the bias (in \%) to the side of the 1st or 2nd stimulus. A negative bias corresponds to a bias to the side of the first stimulus (in patients), whereas a positive bias corresponds to a bias to the side of the second stimulus (in healthy participants).

$17 \mathrm{~ms}$ and is not consciously detected by the participants. After a subsequent delay of between 20 to 10oms, one of the priming frames is filled in and represents the target. Participants have to decide about the right or left location of the filling in and give their response by pressing a right or left response key on the side of the target. At delays of less than 5oms between priming frame presentations and target filling in participants are faster when filling in is in the location of the first frame, consistent with the prior entry effect. At frametarget delays of 10oms, the participants are faster when the filling in is in the location of the second rather than the first frame, consistent with the Simon effect observed in our previous studies. The RT difference is of about $20 \mathrm{~ms}$. This result has been found to be significant in three different groups of 12 healthy participants. RTs in the neutral condition are intermediate between the two asynchronous conditions. These results are consistent with the idea of some kind of move between the stimuli delayed by short asynchronies.

We then adapted our paradigm to answer the critical question whether participants also coded the succession between frames. Accordingly, instead of filling in only one frame, two frames were filled in with an onset asynchrony of 


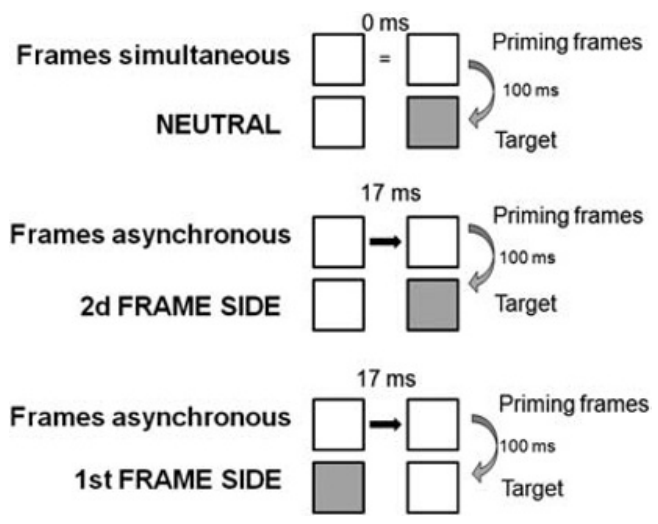

FIGURE 5.5 Illustration of the experimental conditions used in our location priming paradigm. Priming frames were either simultaneous or asynchronous with a SOA of $17 \mathrm{~ms}$ between frames. One of the frames was then filled in and participants had to press a response key to the side of the target. The results correspond to a SOA of 1ooms between prime frames and the target.

10oms, providing a perfectly detectable target order. The order of target filling in was in the same or in the opposite direction compared to priming frame order. This permits a check on whether participants automatically coded the order of the priming frames at an asynchrony of $17 \mathrm{~ms}$. It was expected that if participants coded the priming-frames succession, then this should facilitate perception of the order of target filling in when it matched the order of the frames. However, this is not what was observed: no succession priming was found while instead a facilitation was observed when prime and filling in order were in opposite directions. ${ }^{2}$

The results of the second priming study can be understood easily by taking into account the prior entry effect (Figure 5.6). The previous studies have shown that participants put more emphasis on the location of the second priming frame. This emphasis facilitated the processing of the subsequently

2 The results of the priming studies allow us to eliminate several alternative accounts for the bias to the side of the second stimulus. The first study shows a bias even though the task does not require any judgment of asynchrony or order. This indicates that the bias to the side of the second stimulus does not depend on a particular task-set activation. Additionally, the second study shows that apparent motion does not play a role. A processing of apparent motion between the prime frames should have led to the coding of a succession between the frames and should have enabled a priming of the stimulus order. This was not the case (see also Lalanne et al., 2012c, for a discussion on this point). 


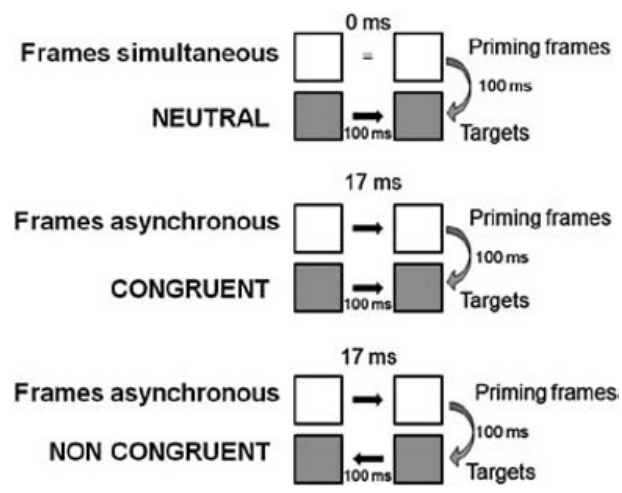

FIGURE 5.6 Illustration of the experimental conditions used in our succession priming paradigm. Priming frames were either simultaneous or asynchronous with a $\mathrm{SOA}$ of $17 \mathrm{~ms}$ between frames. The two frames were then filled in with an asynchrony of $100 \mathrm{~ms}$, with these filled in squares representing the targets. Participants had to press a response key to the side of the 2nd target. The results correspond to a SOA of rooms between prime frames and the targets.

filled in frame in the same location. Like for the prior entry effect, this led to a facilitated response when the first filled in target was in the location of the second priming frame (i.e., when the frame and filling in order was in opposite directions). The RT difference is again of about $20 \mathrm{~ms}$ and has also been reproduced in several groups of 12 healthy participants.

These results, thus, confirm an automatic bias towards the last event in control subjects. They allow us to eliminate alternative explanations of the impact of the short asynchronies, in terms of a coding of motion, direction or task instructions. This has also implications regarding the results observed in patients, inasmuch it suggests the impairments observed in patients are, like for controls, independent from a difficulty in coding motion or in following instructions. The results suggest that the impairment in patients is rather related to a difficulty to follow events over time. Once we have reached this conclusion, however, we can ask about the significance of this impairment. For example, it might be asked how patients are able to follow a stream of incoming information over time in every day life. The impact on duration perception should also be investigated. Inasmuch duration perception relies on the accumulation of information as time moves forward; an inability to follow information over time can be expected to disturb perception duration. However, duration perception concerns time scales that are much longer than those studied here, and theoretical models relating these different time scales are missing (van Wassenhove, 2009; Grondin, 2010). More generally, the consequences of an elementary impairment at moving attention over time, is 
necessarily speculative at this stage. Yet some possibilities seem likely in light of the known impairments described in patients with schizophrenia, and one of them is briefly evoked in the next section.

\section{Possible Impact of Elementary Timing Impairments on Visual Perception}

In every day life, the outer world is usually dynamic rather than static and events succeed each other rapidly. Wind can make tree leaves move, alternately uncovering or occluding visual information. We usually experience no difficulty in distinguishing the tree from the objects located behind it. Yet moving objects like the moving leaves create much ambiguity in the organization of information, and some processing is required in order to attribute elements of information to the right objects. In dynamic environments, this requires discrimination in both space and time: an item has to be precisely focused in time in order to avoid confusion with distracter information, especially if it is displayed in the same location but at a different time. As a matter of fact, the use of masking experiments has shown that patients with schizophrenia have difficulties at distinguishing target information when it is closely preceded or followed by a distracter (Butler et al., 2003; Green and Walker, 1986; Saccuzzo and Braff, 1981). Explanations for this impairment are diverse (Breitmeyer, 1984; Butler et al., 2003; Green et al., 1994; Green et al., 2011; Schuck and Lee, 1989), but the impairment certainly confirms that patients have a difficulty with stimuli shown in close succession. Besides, based on masking experiments, it has been proposed that patients with schizophrenia have a difficulty in the temporal precision of target-directed attention (Granholm et al., 2009; Lalanne et al., 2012a). All in all, temporal difficulties mean heightened difficulties at resolving ambiguities arising in case of dynamic visual information.

\section{$5 \quad$ Relating the Simon Effect with the Sense of Time Continuity}

In summary, it seems that items are distinguished in time at shorter asynchronies than previously believed. However, this is not associated with succession processing, which instead appears to be processed only at larger intervals between events, perhaps associated with the explicit processing of succession. Dissociation between the processing of asynchronies and the sense of succession is frequently reported (reviewed in Wittmann, 2011). What is more surprising is the bias to the side of the second stimulus without any coding of 
succession, although this might be explained relatively easily. Even if the lack of succession coding makes it impossible to tag stimuli as 'first' and 'second', the second one might nevertheless be identified as the latest occurring event. In fact, the bias to the side of the second stimulus in a succession of two suggests the existence of a mechanism putting a priority on the last occurring information. This might be analogous to what happens when attention is driven reflexively by an external event. It is known that attention deploys to novel information, meaning that associated brain mechanisms are designed to continually check for novel information, not only in space but also in time. Feedback loops described in visual perception, predictive coding or forward models might provide neural bases for these mechanisms (Elliott and Müller, 2000; Friston, 2008; Miall et al., 1993; Wolpert et al., 1998). Although the results suggest some kind of prioritization and possibly an involvement of attention, the processes making this prioritization possible are not available to consciousness. Participants do not perceive very short asynchronies, and they put nonetheless more weight on the second and last stimulus. Even if this means the involvement of attention, it cannot be induced by some cognitive expectation. In our priming paradigm, participants have to detect a target and the task does not incite them to expect successive stimuli. This means that some subconscious signal is powerful enough to induce a priming effect and suggests that at least part of the mechanisms making it possible for the last stimulus to be prioritized are automatic in nature.

What is the relationship between the ability to distinguish stimuli at very short asynchronies and the sense of time continuity? In so far as the succession of stimuli is coded only at longer delays, the integration of past, present, and future moments proposed as a mechanism of time continuity should emerge only at large delays. However, what we observe at the shortest delays is an automatic priority for the latest events, possibly sub-tended by mechanisms allowing us to permanently look forward. It might be asked whether this bias to anticipate future events also participates in the sense of continuity, thereby providing an elementary basis for the expectation of what is coming next. Husserl described the concept of a protention, which allows us to anticipate the future during the present time. Although what Husserl describes might be more easily related to conscious phenomena taking place at larger time scales, the bias towards the latest stimulus might nonetheless be considered as an elementary mechanism subserving protention.

Related to that, it can be asked whether patients' disrupted sense of time continuity is related to their difficulty at assigning priority to the latest occurring events. It certainly reminds of the observation of Minkowski (1933), who described a loss of life force in patients with schizophrenia. 
More concretely, it might be asked how patients perceive a rapid succession. If they cannot move easily from the first to the second event, do they miss information in everyday life? How is this related with the way they perceive duration (Allman and Meck, 2012; Davalos et al., 2005; Elvevåg et al., 2004; Volz et al., 2001) or time actions (Carroll et al., 2009; Delevoye-Turrell et al., 2012; Turgeon et al., 2012)?

\section{Conclusion}

Although many questions remain, our results show that information is not fused in time at very short asynchronies, either in healthy participants or in patients. Our results also indicate that healthy participants move in time very rapidly between succeeding events and that this capability is impaired in patients. These observations may mean new additional mechanisms underlying the sense of time continuity. It remains to be investigated whether and how these mechanisms are really involved in the sense of time continuity, and how the impairments of these mechanisms impact cognitive functions and clinical symptoms in schizophrenia.

\section{References}

Allman, Melissa J., and Warren H. Meck. 2012. "Pathophysiological Distorsions in Time Perception and Timed Performance" Brain 135: 656-77.

Andreasen, Nancy C. 1999. “A Unitary Model of Schizophrenia: Bleuler's 'Fragmented Phrene' as Schizencephaly" Archives of General Psychiatry 56 : 781-7.

Brecher, Gerhard A. 1932. "Die Entstehung und biologische Bedeutung der subjektiven Zeiteinheit-des Momentes" Zeitschrift für Vergleichende Physiologie 18: 204-43.

Breitmeyer, Bruno G. 1984. Visual Masking: An Integrative Approach New York: Oxford University Press.

Butler, Pamela D. et al. 2003. "Visual Backward-Masking Deficits in Schizophrenia: Relationship to Visual Pathway Function and Symptomatology" Schizophrenia Research 1: 199-209.

Carroll, Christine A. et al. 2009. "Timing Dysfunctions in Schizophrenia" Brain Cognition 71: $345-53$.

Chapman, James. 1966. "The Early Symptoms of Schizophrenia" British Journal of Psychiatry 112: 225-51.

Dainton, Barry. 2010. "Temporal Consciousness." In The Stanford Encyclopedia of Philosophy, edited by Edward N. Zalta. Available at <http://plato.stanford.edu/ archives/fall2010/entries/consciousness-temporal>. 
Davalos, Deana B., Michael A. Kisley, and Robert Freedman. 2005. "Behavioral and Electrophysiological Indices of Temporal Processing Dysfunction in Schizophrenia" The Journal of Neuropsychiatry and Clinical Neurosciences 17:517-25.

David, Anthony S. 1993. "Callosal Transfer in Schizophrenia: Too Much or Too Little?" Journal of Abnormal Psychology 102: 573-9.

Del Cul, Antoine, Stanislas Dehaene, and Marion Leboyer. 20o6. "Preserved Subliminal Processing and Impaired Conscious Access in Schizophrenia" Archives in General Psychiatry 63: 1313-23.

Delevoye-Turrell, Yvonne, Hélène Wilquin, and Anne Giersch. 2012. “A Ticking Clock for the Production of Sequential Actions: Where Does the Problem Lie in Schizophrenia?" Schizophrenia Research 135: 51-4.

Delevoye-Turrell, Yvonne, Anne Giersch, and Jean-Marie Danion. 2003. "Abnormal Sequencing of Motor Actions in Patients with Schizophrenia: Evidence from Grip Force Adjustments during Object Manipulation" American Journal of Psychiatry 16o: 134-41.

Elliott, Mark A. 2005. "Temporal Tolerance Circumscribed" Visual Cognition 12: $655^{-61 .}$

Elliott, Mark A., and Hermann J. Müller. 200o. "Evidence for a 40-Hz Oscillatory ShortTerm Visual Memory Revealed by Human Reaction Time Measurements” Journal of Experimental Psychology: Learning, Memory and Cognition 26: 703-18.

Elliott, Mark A., Zhuanghua Shi, and Sean D. Kelly. 20o6. "A Moment to Reflect Upon Perceptual Synchrony." Journal of Cognitive Neuroscience 18: 1663-5.

Elliott, Mark A., Zhuanghua Shi, and Fatma Sürer. 2007. "The Effects of Subthreshold Synchrony on the Perception of Simultaneity" Psychological Research 71: 687-93.

Elvevåg, Brita et al. 2004. "Identification of Tone Duration, Line Length, and Letter Position: An Experimental Approach to Timing and Working Memory Deficits in Schizophrenia" Journal of Abnormal Psychology 113: 509-21.

Exner, Sigmund. 1875. "Experimentelle Untersuchungen der einfachsten psychischen Processe" Pflügers Archives 11: 403-32.

Foucher, Jack R. et al. 2007. "Low Time Resolution in Schizophrenia Lengthened Windows of Simultaneity for Visual, Auditory and Bimodal Stimuli" Schizophrenia Research 97: 118-27.

Friston, Karl. 2008. "Hierarchical Models in the Brain" PLos Computational Biology, 4: e1000209.

Fuchs, Thomas. 2007. "The Temporal Structure of Intentionality and Its Disturbance in Schizophrenia" Psychopathology 40: 229-35.

Giersch, Anne et al. 2009. "Extended Visual Simultaneity Thresholds in Patients with Schizophrenia" Schizophrenia Bulletin 35: 816-25.

Giersch, Anne, and Virginie Rhein. 2008. "Lack of Flexibility in Visual Grouping in Patients with Schizophrenia" Journal of Abnormal Psychology 117: 132-42. 
Granholm, Eric, Scott C. Fish, and Steven P. Verney. 2009. "Pupillometric Measures of Attentional Allocation to Target and Mask Processing on the Backward Masking Task in Schizophrenia" Psychophysiology 46: 510-20.

Green, David M., and John A. Swets. 1974. Signal Detection Theory and Psychophysics Melbourne, Florida: Robert E. Krieger Publishing Co.

Green, Michael F. and Elaine Walker. 1986. "Symptom Correlates of Vulnerability Backward Masking in Schizophrenia” American Journal of Psychiatry 143: 181-6.

Green, Michael F., Keith H. Nuechterlein, and Jim Mintz. 1994. "Backward Masking in Schizophrenia and Mania. II. Specifying the Visual Channels" Archives of General Psychiatry 51: 945-51.

Green, Michael F. et al. 2011. "Visual Masking in Schizophrenia: Overview and Theoretical Implications" Schizophrenia Bulletin 37: 700-8.

Grondin, Simon. 2010. "Timing and Time Perception: A Review of Recent Behavioral and Neuroscience Findings and Theoretical Directions" Attention Perception and Psychophysics 272: 561-82.

Holzman, Philip S. 2000. "Eye Movements and the Search for the Essence of Schizophrenia" Brain Research: Brain Research Reviews 31: 350-6.

Hommel, Bernhard. 2011a. "The Simon Effect as a Tool and Heuristic" Acta Psychologica 136: 189-202.

Hommel, Bernhard. 2011b. "Attention and Spatial Stimulus Coding in the Simon Task: A Rejoinder to Van Der Lubbe and Abrahamse (2010)" Acta Psychologica 136: 265-68.

Husserl, Edmund. 1893-1917. On the Phenomenology of the Consciousness of Internal Time (1893-1917) Brough JB, trans-ed. Dordrecht, Boston: Kluwer Academic Publishers, 1991.

James, William. 1896. "The Perception of Time” In The Principles of Psychology, Chapter $X V$, London: McMillan.

Knöchel, Christian et al. 2012. "Interhemispheric Hypoconnectivity in Schizophrenia: Fiber Integrity and Volume Differences of the Corpus Callosum in Patients and Unaffected Relatives" Neuroimage 59: 926-34.

Lalanne, Léon. 1876. "Sur la durée de la sensation tactile" Note des Comptes rendus de l'Académie des Sciences de Paris, 1314-6.

Lalanne, Laurence et al. 2012a. "Attention and Masking in Schizophrenia" Biological Psychiatry 71: 162-8.

Lalanne, Laurence, Mitsouko Van Assche, and Anne Giersch. 2012b. "When Predictive Mechanisms Go Wrong: Disordered Visual Synchrony Thresholds in Schizophrenia" Schizophrenia Bulletin 38: 506-13.

Lalanne, Laurence et al. 2012c. "Looking Forward: An Impaired Ability in Patients with Schizophrenia?" Neuropsychologia 50: 2736-44.

Martin, Brice et al. (2013). "Temporal Event Structure and Timing in Schizophrenia: Preserved Binding in a Longer 'now'” Neuropsychologia 51: 358-371. 
Miall, R. et al. 1993. "Is the Cerebellum a Smith Predictor?" Journal of motor behavior 25: 203-16.

Minkowski, Eugène. 1933. Le temps vécu Presses Universitaires de France: Paris.

Mohr, Bettina et al. 2008. "Hemispheric Cooperation-a Crucial Factor in Schizophrenia? Neurophysiological Evidence" NeuroImage 41: 1102-10.

Nicol, Jeffrey R., and David I. Shore. 2007. "Perceptual Grouping Impairs Temporal Resolution" Experimental Brain Research 183: 141-8.

Phillips, Mary L., and Anthony S. Davis. 1994. "Understanding the Symptoms of Schizophrenia Using Visual Scan Paths" British Journal of Psychiatry 165: 673-5.

Pöppel, Ernst. 1997. "A Hierarchical Model of Temporal Perception" Trends in Cognitive Science 1: 56-61.

Pöppel, Ernst. 2009. "Pre-Semantically Defined Window for Cognitive Processing" Philosophical Transactions of the Royal Society B Biological Sciences, 364: 1887-96.

Saccuzzo, Dennis P., and David L. Braff. 1981. "Early Information Processing Deficit in Schizophrenia News Findings Using Schizophrenic Patients Centrally, Not Peripherally" Archives of General Psychiatry, 38: 175-9.

Schmidt, Heike et al. 2011. "Low-Level Temporal Coding Impairments in Psychosis: Preliminary Findings and Recommendations for Further Studies" Journal of Abnormal Psychology 120: 476-82.

Schwartz, Barry D., Daniel K. Winstead, and W.G. Walker. 1984. "A Corpus Callosal Deficit in Sequencial Analysis by Schizophrenics" Biological Psychiatry 19: 1667-76. Schuck, John R., and Richard G. Lee. 1989. "Backward Masking, Information Processing, and Schizophrenia" Schizophrenia Bulletin 15: 491-500.

Silverstein, Steven M., and Brian P. Keane. 2011. "Perceptual Organization Impairment in Schizophrenia and Associated Brain Mechanisms: Review of Research from 2005 to 2010" Schizophrenia Bulletin 37: 690-9.

Spence, Charles, and Cesare Parise. 2010. "Prior-Entry: A Review" Consciousness and Cognition 19: 364-79.

Szelag, Elzbieta et al. 2004. "Individual Differences in Temporal Information Processing in Humans" Acta Neurobiologica Experimentalis 64: 349-66.

Trillenberg, Peter, Rebekka Lencer, and Wolfgang Heide. 2004. "Eye Movements and Psychiatric Disease" Current Opinion in Neurology 17: 43-7.

Turgeon, Martine et al. 2012. "Impaired Predictive Timing with Spared Time Interval Production in Individuals with Schizophrenia" Psychiatry Research 197: 13-8.

Uhlhaas, Peter, and Wolf Singer. 2010. "Abnormal Neural Oscillations and Synchrony in Schizophrenia" Nature Reviews Neuroscience 11: 100-13.

Van Assche, Mitsouko, and Anne Giersch. 2011. "Visual Organization Processes in Schizophrenia" Schizophrenia Bulletin 37: 394-404.

Van der Lubbe, Rob H.J., and Elger L. Abrahamse. 2011. "The Premotor Theory of Attention and the Simon Effect" Acta Psychologica 136: 259-64. 
Van Wassenhove, Virginie. 20og. "Minding Time in an Amodal Representational Space" Philosophical Transactions of the Royal Society B Biological Sciences 364: 1815-30.

Varela, Francisco J. (1999). "The Specious Present: A Neurophenomenology of Time Consciousness" In Naturalizing Phenomenology. Issues in ContemporaryPhenomenology and Cognitive Science, edited by Jean Petitot, 266-329. Stanford: Stanford University Press.

Vogeley, Kai, and Christian Kupke. 2007. "Disturbances of Time Consciousness from a Phenomenological and Neuroscientific Perspective" Schizophrenia Bulletin 33: $142-56$.

Volz, Hans-Peter et al. 2001. "Time Estimation in Schizophrenia: An fMRI Study at Adjusted Levels of Difficulty" Neuroreport 12: 313-6.

Von Baer, Karl Ernst (1864). "Welche Auffassung der lebenden Natur ist die richtige? Und wie ist diese Auffassung auf die Entomologie anzuwenden?" In Reden, gehalten in wissenschaftlichen Versammlungen une kleinere Aufsätze vermischten Inhalt, edited by Karl Ernst von Baer, 237-84. St-Petersburg, Russia: H. Schmitzdorf.

Wittmann, Marc. 2011. "Moments in Time" Frontiers in Integrative Neuroscience 5(66): 1-9.

Wolpert, Daniel M., R. Chris Miall, and Mitsuo Kawato. 1998. "Internal Models in the Cerebellum" Trends in Cognitive Science 2: 338-47. 